\title{
Parental-origin-determination fluorescence in situ hybridization distinguishes homologous human chromosomes on a single-cell level
}

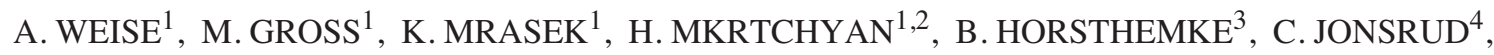 \\ F. VON EGGELING ${ }^{1}$, S. HINREINER ${ }^{1}$, V. WITTHUHN ${ }^{1}$, U. CLAUSSEN $^{1}$ and T. LIEHR $^{1}$
}

\author{
${ }^{1}$ Institute of Human Genetics and Anthropology, Jena, Germany; ${ }^{2}$ Department of Genetic and Laboratory of Cytogenetics, \\ Jerewan, Armenia; ${ }^{3}$ Institute of Human Genetics, Essen, Germany; ${ }^{4}$ Department of Medical Genetics, Troms $\varnothing$, Norway
}

Received September 24, 2007; Accepted November 5, 2007

\begin{abstract}
The differentiation of homologous chromosomes as well as their parental origin can presently be conducted and determined exclusively by molecular genetic methods using microsatellite or SNP analysis. Only in exceptional cases is a distinction on a single-cell level possible, e.g. due to variations within the heterochromatic regions of chromosomes $1,9,16$ and $\mathrm{Y}$ or the $\mathrm{p}$-arms of the acrocentric chromosomes. In the absence of such polymorphisms, an individual distinction of the homologous chromosomes is not currently possible. Consequently, various questions of scientific and diagnostic relevance are unable to be answered. Based on the recently detected large-scale copy-number variations (LCV) or copynumber polymorphisms (CNP) spanning up to several megabase pairs of DNA, in this study, a molecular cytogenetic technique for the inter-individual differentiation of homologous chromosomes called parental-origin-determination fluorescence in situ hybridization (pod-FISH) is presented. All human chromosomes were covered with $225 \mathrm{LCV}$ and/or CNP-specific BAC probes, and one- to five-color chromosome-specific pod-FISH sets were created, evaluated and optimized. We demonstrated that pod-FISH is suitable for single-cell analysis of uniparental disomy (UDP) in clinical cases such as Prader-Willi syndrome caused by maternal UPD. A rare clinical case with a mosaic form of a genome-wide isodisomy was used to determine the detection limits of podFISH. Additionally we analyzed the informativeness of conventional microsatellite analysis for the first time and compared the results to pod-FISH. With this new possibility
\end{abstract}

Correspondence to: Dr Anja Weise, Institute of Human Genetics and Anthropology, D-07740 Jena, Germany

E-mail: aweise@mti.uni-jena.de

Key words: parental-origin-determination fluorescence in situ hybridization, homologous chromosomes, uniparental disomy, large-scale copy-number variations, copy-number polymorphisms, polymorphism to study the parental origin of individual human chromosomes on a single-cell level, new doors for diagnostic and basic research are opened.

\section{Introduction}

Considering the molecular genetic level of the genome, there is a genetic variation, up to $0.1 \%$ of the DNA sequence, between any two human individuals. These differences include single-nucleotide polymorphisms (SNP), which appear in every thousand base pairs on average and are located in or outside coding regions (1), small insertiondeletion polymorphisms (INDEL) and non-coding polymorphisms like mini- and microsatellites (2). Although these DNA variations affect alleles on homologous chromosomes they cannot be used to distinguish chromosomes on a singlecell level. Differentiation of the parental origin of homologous sequences is therefore currently possible only by molecular genetic methods, such as using DNA isolated from a mixture of many different single cells, followed by approaches such as microsatellite analysis (3) or methylationsensitive PCR (4). In contrast, conventional cytogenetics allows a differentiation with respect to maternal or paternal origin on a single-cell level, but only for a specific subset of human chromosomes and only in exceptional cases (5). This can be conducted based on size variations of the heterochromatic regions of chromosomes 1,9,16 and Y, or of the short arms of acrocentric chromosomes, inversion polymorphisms for chromosomes 2 and $9(6,7)$ or even less frequently, if different dimensions of centromeric heterochromatin (cenh+ variants) are observed (8). In the absence of such microscopically visible polymorphisms a discrimination between human homologous chromosomes is impossible on a singlecell level (Fig. 1). Consequently, a number of questions of scientific and diagnostic relevance remain unanswered.

The picture of the human genome was remarkably changed and extended by a new kind of polymorphism discovered three years ago, called large-scale copy-number variations (LCV) (9) or copy-number polymorphisms (CNP) (10). These variations were found by DNA microarray technology and include hundreds of previously undetected structural variants in the human genome such as deletions, gains and 


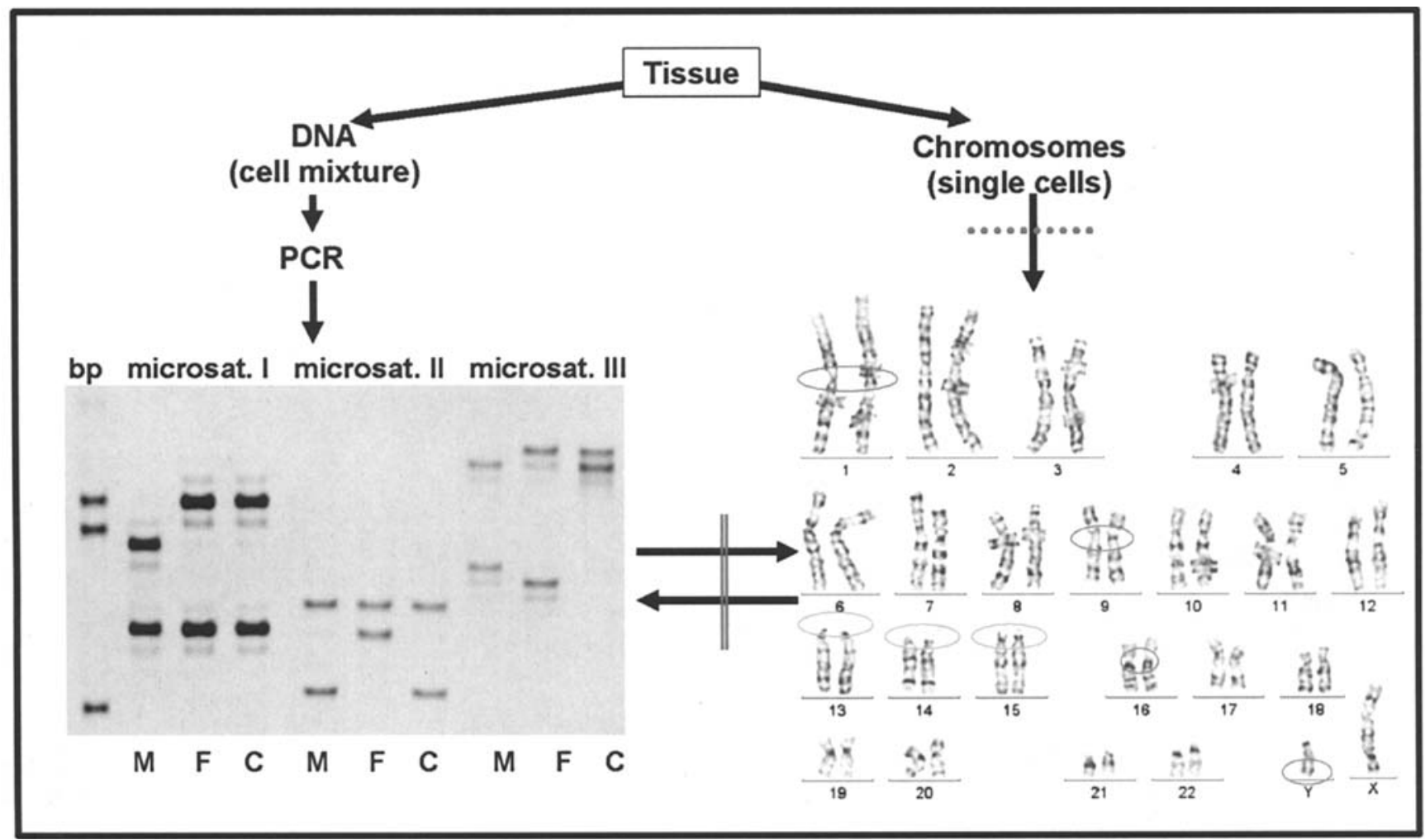

Figure 1. Two methods for distinguishing the parental origin of alleles are (left) by molecular genetic methods such as microsatellites (M, mother; F, father; $\mathrm{C}$, child) based on isolated DNA from a cell mixture and (right) on a single-cell level by chromosomes that show cytogenetic polymorphisms in certain individuals and chromosomes. Yet, there is no possibility to connect the molecular genetic allele information with the homologous chromosomes and visa versa.

inversions. Surprisingly, LCV/CNP can have sizes of ten to several hundred thousand base pairs and are located in euchromatic regions all over the genome. It is expected that these large scale variations can directly affect gene dosage and therefore contribute essentially to phenotypic variation in humans (1). Currently (July 2007), there are 3643 reported structural variations (9-20) that have been collected in the database of genomic variants (http://projects.tcag.ca/ variation/).

Concerning the huge size of these newly described structural variants it is currently possible to connect the molecular genetic level of DNA polymorphisms with microscopically visible homologous chromosomes. Moreover, they may be used for distinguishing cytogenetically identical homologous chromosomes. Utilizing this advantage, we describe for the first time the discrimination of homologous human chromosomes based on DNA sequence polymorphisms. This was achieved by a special fluorescence in situ hybridization (FISH) technique; the so-called parental-origindetermination FISH (pod-FISH). The usefulness and feasibility of this new approach is demonstrated, its efficiency will be compared to conventional microsatellite analysis and prospects for future applications are discussed.

\section{Materials and methods}

Cells. Molecular cytogenetic studies were performed on peripheral blood lymphocytes. The evaluation of pod-FISH probes was performed on chromosomes from one healthy proband. Chromosome-specific pod-FISH sets for chromosomes 1,9 and 16 were tested on five probands showing a heterochromatin enlargement in one homologous chromosome (as described above). pod-FISH sets for all chromosomes were tested on a rare clinical case of a mosaic form of a genome-wide isodisomy. Chromosome preparations were conducted according to standard techniques (21).

Molecular cytogenetics. Two hundred and twenty-five polymorphic regions were selected which had a size $>150 \mathrm{~kb}$, that were reported in more than one person or study and that were reported for loss from the database of genomic variants (http://projects. tcag.ca/variation/). BAC clones were purchased from the Children's Hospital Oakland Research Institute (CHORI), Oakland, CA, USA or kindly provided by the Sanger Center. A list of all BAC clones is provided in Table I. All BAC DNA was isolated, PCR-amplified and labeled as described (22). Single and multicolor fluorescence in situ hybridization (FISH) techniques were performed according to published protocols $(22,23)$.

Microsatellite analysis. Microsatellite studies were conducted in 182 UPD analyses on the basis of 299 different microsatellites (data not shown). Therefore genomic DNA was isolated from the lymphocytes of the patients, and PCR was performed as described in (24).

\section{Results and discussion}

Selection of probes and analysis of pod-FISH signals. Two hundred and twenty-five of over 3643 polymorphic loci were selected from the database of genomic variants. These were chosen, as they were reported to be frequently deleted and 
Table I. Overview of BAC clones used for pod-FISH based on NCBI build 36.2.

\begin{tabular}{|c|c|c|c|c|c|c|}
\hline Chr & Band & VL & $\mathrm{BAC}$ & $\mathrm{AC}$ & Start kb & End $\mathrm{kb}$ \\
\hline 1 & p36.33 & 566 & RP11-430E19 & AQ552337 & 18 & 167 \\
\hline 1 & p36.33 & 2 & RP1-283E3 & AL031282 & 1609 & 1720 \\
\hline 1 & p36.22 & 567 & RP4-636F13 & AL109757 & 12351 & 12406 \\
\hline 1 & p36.13 & 5 & RP1-163M9 & AL021920 & 16880 & 16998 \\
\hline 1 & p36.12 & 2050 & RP11-69E9 & AQ267525 & 22955 & 23136 \\
\hline 1 & p32.2 & 9 & RP6-65F20 & AL138779 & 57301 & 57385 \\
\hline 1 & $\mathrm{p} 31.2$ & 10 & RP11-131O15 & AL358512 & 67446 & 67607 \\
\hline 1 & $\mathrm{p} 21.1$ & 326 & RP11-79H19 & AQ284254 & 102405 & 102563 \\
\hline 1 & $\mathrm{p} 21.1$ & 13 & RP11-259N12 & AL590104 & 103956 & 104113 \\
\hline 1 & p21 & 13 & RP11-508C1 ${ }^{a}$ & AL513482 & 103921 & 104016 \\
\hline 1 & p13.3 & 2051 & RP11-242D10 & AQ488595 & 108594 & 108594 \\
\hline 1 & $\mathrm{q} 21.1$ & 1472 & RP11-415J5 & AQ547195 & 143852 & 143853 \\
\hline 1 & q21.1 & 18 & RP11-458D21 & AL592307 & 143900 & 143901 \\
\hline 1 & q21.1 & 2053 & RP11-18E7 & B85485 & 146578 & 146579 \\
\hline 1 & $\mathrm{q} 21.1-\mathrm{q} 21.2$ & 351 & RP11-241H1 & AQ481397 & 148067 & 148068 \\
\hline 1 & $\mathrm{q} 25.2$ & 21 & RP11-415M14 & AL162736 & 174297 & 174457 \\
\hline 1 & q31 & 568 & RP11-109P13 & BX248415 & 195063 & 195222 \\
\hline 1 & $\mathrm{q} 31.3$ & 2054 & RP11-179H2 & AQ419857 & 194526 & 194686 \\
\hline 1 & $\mathrm{q} 42.2$ & 22 & RP5-1016N21 & AL139342 & 231322 & 231474 \\
\hline 1 & $\mathrm{q} 44$ & 24 & RP11-438F14a & AC098483 & 245013 & 245191 \\
\hline 2 & p22.3 & 25 & RP11-119B15 & $\mathrm{AC} 068274$ & 35718 & 35886 \\
\hline 2 & p21.1 & 642 & RP11-130P22 & AC016696 & 46251 & 46406 \\
\hline 2 & p15 & 27 & RP11-355B11 & AC016727 & 61513 & 61673 \\
\hline 2 & p12 & 29 & RP11-398N13a & AC114767 & 82772 & 82779 \\
\hline 2 & $\mathrm{p} 11.2$ & 31 & RP11-685N3a & AC096767 & 89040 & 89182 \\
\hline 2 & $\mathrm{p} 11.2$ & 29 & RP11-495B16 & AC109638 & 82554 & 82717 \\
\hline 2 & $\mathrm{q} 21$ & 36 & RP11-32C20 & AC108865 & 130418 & 130583 \\
\hline 2 & $\mathrm{q} 21.1$ & 37 & RP11-89B17 & AQ283656 & 131997 & 132171 \\
\hline 2 & $\mathrm{q} 31.1$ & 40 & RP11-80D14 & AQ283974 & 170976 & 171138 \\
\hline 2 & $\mathrm{q} 35$ & 44 & RP11-316O14 & AC053503 & 219915 & 220099 \\
\hline 2 & $\mathrm{q} 37$ & 46 & RP11-341N2 & AC093642 & 242709 & 242710 \\
\hline 3 & p26 & 779 & RP11-151A4 & AQ377442 & 794 & 795 \\
\hline 3 & $\mathrm{p} 25.1$ & 645 & RP11-57D6 & AQ116103 & 13144 & 13315 \\
\hline 3 & $\mathrm{p} 21$ & 47 & RP11-34D21 & AQ046388 & 60224 & 60224 \\
\hline 3 & $\mathrm{p} 12$ & 377 & RP11-652K20 & AQ407100 & 84963 & 85121 \\
\hline 3 & q13.33 & 49 & RP11-169N13 & AC069444 & 120926 & 121091 \\
\hline 3 & q26.1 & 52 & RP11-79F11 & AQ284158 & 165317 & 165467 \\
\hline 3 & q26.1 & 53 & RP11-91B7 & AQ283223 & 168999 & 169156 \\
\hline 3 & q26.32 & 54 & RP11-114M1 & AC026355 & 178755 & 178912 \\
\hline 3 & q29 & 2056 & RP11-245H8 & AQ489110 & 195974 & 196140 \\
\hline 3 & q29 & 56 & RP11-1112010 & AQ747384 & 196903 & 197039 \\
\hline 4 & p16.3 & 2057 & RP11-349C22 & AQ543331 & 248 & 418 \\
\hline 4 & p16.1 & 2058 & RP11-261G12 & AQ482925 & 6262 & 6437 \\
\hline 4 & p16.3 & 61 & RP11-125L6 & AQ344959 & 8950 & 8989 \\
\hline 4 & p15.1 & 63 & RP11-81N11 & AQ281893 & 34453 & 34603 \\
\hline 4 & $\mathrm{p} 12$ & 572 & RP11-238D1 & AZ521050 & 45621 & 45630 \\
\hline 4 & $\mathrm{q} 13.2$ & 2060 & RP11-279G22 & AQ507098 & 68501 & 68658 \\
\hline 4 & q13.3 & 64 & RP11-121P15 & AQ351558 & 71415 & 71415 \\
\hline 4 & q25 & 69 & RP11-18D18 & B82859 & 112484 & 112501 \\
\hline 4 & q31.21 & 1651 & RP11-412J20 & AQ536199 & 144877 & 145027 \\
\hline 4 & q32.2 & 73 & RP11-1003 & AC096717 & 162954 & 163127 \\
\hline 4 & q33 & 75 & RP11-90E13 & AQ281517 & 171360 & 171524 \\
\hline 5 & p15.33 & 80 & RP11-812N8 & AQ722828 & 780 & 879 \\
\hline 5 & p15.1 & 85 & RP11-88L18 & $A Q 281510$ & 17465 & 17630 \\
\hline 5 & p14.3 & 398 & RP11-167E4 & AQ382457 & 21493 & 21635 \\
\hline 5 & q13.2 & 89 & RP11-551B22 & AC012361 & 70330 & 70517 \\
\hline
\end{tabular}


Table I. Continued.

\begin{tabular}{|c|c|c|c|c|c|c|}
\hline Chr & Band & VL & $\mathrm{BAC}$ & $\mathrm{AC}$ & Start kb & End $\mathrm{kb}$ \\
\hline 5 & q14.1 & 90 & RP11-90A9 & AQ286808 & 79882 & 80063 \\
\hline 5 & $\mathrm{q} 21.1$ & 2061 & RP11-106M6 & AQ317103 & 99184 & 99367 \\
\hline 5 & $\mathrm{q} 21$ & 577 & RP11-346N7 & AQ531580 & 99628 & 99629 \\
\hline 5 & $\mathrm{q} 31.3$ & 93 & RP11-55M16 & AQ082942 & 141030 & 141195 \\
\hline 5 & q35.2 & 2062 & RP11-259L15 & AQ482916 & 174876 & 175053 \\
\hline 5 & $\mathrm{q} 35.3$ & 2063 & RP11-235D6 ${ }^{\mathrm{b}}$ & n.a. & n.a. & n.a. \\
\hline 5 & $\mathrm{q} 35.3$ & 2063 & RP11-516K1 & BH634779 & 180335 & 180335 \\
\hline 6 & $\mathrm{p} 25.3$ & 95 & RP3-416J7 & AL035696 & 89 & 214 \\
\hline 6 & p22.1 & 578 & RP11- 111A4 & AQ322113 & 26949 & 27107 \\
\hline 6 & p21.31 & 102 & RP3-368C2 & AL395494 & 35624 & 35740 \\
\hline 6 & p21.1 & 104 & RP3-447E21 & AL050336 & 46035 & 46196 \\
\hline 6 & $\mathrm{p} 12.3$ & 579 & RP11-11J8 & B74900 & 47414 & 47569 \\
\hline 6 & q12 & 110 & RP3-442I1 & AL078597 & 65020 & 65158 \\
\hline 6 & q12 & 112 & RP11-80L16 & AQ284167 & 67140 & 67141 \\
\hline 6 & q14.1 & 116 & RP11-897A20 & AQ668040 & 79105 & 79106 \\
\hline 6 & q16.3 & 580 & RP3-399E4 ${ }^{a}$ & AL121948 & 100935 & 100955 \\
\hline 6 & q21 & 118 & RP1-70A9 & AL121788 & 109965 & 110127 \\
\hline 6 & $q 24.3$ & 122 & RP1-69B13 & AL035698 & 146560 & 146865 \\
\hline 6 & q27 & 2064 & RP11-16010 & B76754 & 167420 & 167579 \\
\hline 7 & p22 & 1704 & RP11-460K7 & AQ633429 & 7227 & 7420 \\
\hline 7 & $\mathrm{q} 21.1$ & 124 & RP11-79G16 & AQ581376 & 13786 & 13936 \\
\hline 7 & $\mathrm{p} 21.1$ & 126 & RP11-316L18 & AQ507817 & 19069 & 19233 \\
\hline 7 & q11.1 & 339 & RP11-144H20 & AC019063 & 61413 & 61600 \\
\hline 7 & q11.22 & 2065 & RP11-118D11 & AQ347920 & 66515 & 66674 \\
\hline 7 & $\mathrm{q} 11.23$ & 321 & RP11-159N6 & AQ374085 & 72026 & 72128 \\
\hline 7 & q21.11 & 130 & RP11-90N9 & AQ284547 & 83058 & 83206 \\
\hline 7 & q22.1 & 2066 & RP11- 204M9 & AQ414874 & 99410 & 99593 \\
\hline 7 & $\mathrm{q} 22$ & 430 & $\mathrm{RP} 11-188 \mathrm{C} 21^{\mathrm{b}}$ & AQ417326 & 101711 & 101750 \\
\hline 7 & $\mathrm{q} 22$ & 132 & RP11-577H5 & AC105052 & 101820 & 102007 \\
\hline 7 & $\mathrm{q} 31.1$ & 133 & RP11-89O20 & AQ283596 & 112297 & 112436 \\
\hline 7 & q34 & 585 & RP11-45N9 & AQ195715 & 143536 & 143690 \\
\hline 7 & q35 & 135 & RP4-669B10 & $\mathrm{AC} 004853$ & 143053 & 143082 \\
\hline 8 & $\mathrm{p} 23.3$ & 140 & RP11-159F11 & AQ372530 & 2215 & 2435 \\
\hline 8 & p23 & 440 & RP11-774P7 & AQ522799 & 7732 & 7917 \\
\hline 8 & p23.1 & 440 & RP11-52B19 & AQ115704 & 7873 & 7873 \\
\hline 8 & p22 & 1758 & RP11-366J3 & AQ527596 & 14892 & 14893 \\
\hline 8 & p22 & 144 & RP11-90I3 & AQ281649 & 16716 & 16894 \\
\hline 8 & $\mathrm{p} 21.3$ & 2068 & RP11-459H21 & AQ580929 & 21181 & 21389 \\
\hline 8 & $\mathrm{q} 21.2$ & 445 & RP11-96G1 & AC023390 & 86851 & 86956 \\
\hline 8 & $\mathrm{q} 22.2$ & 151 & RP11-959D4 & AQ742248 & 100283 & 100284 \\
\hline 8 & $\mathrm{q} 24.22$ & 155 & RP11-21H16 & A084813 & 135975 & 136155 \\
\hline 9 & p24.3 & 158 & RP11-130C19 & AL136979 & 614 & 843 \\
\hline 9 & p22.2 & 160 & RP11-340N12 & AL354711 & 17136 & 17299 \\
\hline 9 & p12 & 594 & $\mathrm{RP} 11-429 \mathrm{~F} 11^{\mathrm{a}}$ & AQ550525 & 45232 & 45382 \\
\hline 9 & p12 & 161 & RP11-93P10 & AQ312901 & 65762 & 65932 \\
\hline 9 & p11.2 & 2071 & RP11-316A6 & AQ539585 & 42838 & 43023 \\
\hline 9 & $\mathrm{p} 11.2$ & 594 & RP11-343E15 & AQ537613 & 45835 & 45836 \\
\hline 9 & $\mathrm{p} 11.2$ & 592 & RP11-433F23 & AQ581745 & 45945 & 46106 \\
\hline 9 & q12 & 595 & RP11-452D2 & AL591438 & 67803 & 67803 \\
\hline 9 & q12 & 2072 & RP11-194F8 & AQ413960 & 68095 & 70113 \\
\hline 9 & $\mathrm{q} 21.31$ & 165 & RP11-79G7 & AQ283445 & 81540 & 81718 \\
\hline 9 & q32 & 169 & RP11-9H12 & B72555 & 114865 & 115042 \\
\hline 9 & q33.1 & 246 & RP11-80J8b & n.a. & n.a. & n.a. \\
\hline 9 & $\mathrm{q} 34.3$ & 171 & RP11-413M3 & AL592301 & 136526 & 136715 \\
\hline 10 & q11.22 & 174 & RP11-314P12 & AL390716 & 46487 & 46562 \\
\hline 10 & q22.3 & 176 & RP11-19C18 & B82956 & 78621 & 78771 \\
\hline
\end{tabular}


Table I. Continued.

\begin{tabular}{|c|c|c|c|c|c|c|}
\hline Chr & Band & VL & $\mathrm{BAC}$ & $\mathrm{AC}$ & Start kb & End $\mathrm{kb}$ \\
\hline 10 & $\mathrm{q} 22.3$ & 2075 & RP11-259A6 & AQ485496 & 80085 & 80242 \\
\hline 10 & $\mathrm{q} 22.3$ & 598 & RP11-136P13 & AQ346822 & 81551 & 81589 \\
\hline 10 & $q 23.1$ & 2077 & RP11-137H2 & AQ382286 & 82157 & 82319 \\
\hline 10 & $\mathrm{q} 23.1$ & 599 & RP11-80I7 & AQ281216 & 82383 & 82540 \\
\hline 10 & q26 & 340 & RP11-108K14 & AL161645 & 135079 & 135240 \\
\hline 11 & p15 & 601 & RP11-1151C19 & AC1393737 & 4143 & 4293 \\
\hline 11 & p15 & 181 & RP11-168C2a & AC015700 & 10078 & 10257 \\
\hline 11 & p15.1 & 2078 & RP1-239B22 ${ }^{\text {a }}$ & AC124798 & 17328 & 17469 \\
\hline 11 & p14.3-p15.1 & 2079 & RP11-261I18 & AQ483542 & 21513 & 21682 \\
\hline 11 & $\mathrm{p} 11.2$ & 185 & RP11-79A4 & AQ282359 & 48644 & 48801 \\
\hline 11 & q11 & 186 & RP11-380022 & AQ535337 & 55216 & 55378 \\
\hline 11 & q12.3 & 189 & RP11-49D19 & AQ052920 & 62271 & 62448 \\
\hline 11 & q13.2 & 2080 & RP11-280I11 & AQ508928 & 67272 & 67273 \\
\hline 11 & q14.1 & 1860 & RP11-19P3 & B88382 & 84347 & 84518 \\
\hline 11 & q14.1 & 1860 & RP11-19P3 & B88382 & 84348 & 84517 \\
\hline 11 & $\mathrm{q} 22.2$ & 195 & RP11-33F6 & AQ044970 & 102439 & 102497 \\
\hline 11 & q23.3 & 199 & RP11-356E17 & AQ535047 & 115831 & 116041 \\
\hline 12 & p13.33 & 201 & RP11-543P15 & AC005912 & 3099 & 3265 \\
\hline 12 & p12.1 & 205 & RP11-12D15 & AC007544 & 22210 & 22369 \\
\hline 12 & $q 21.32$ & 209 & RP11-900F13 & AC024941 & 87374 & 87547 \\
\hline 12 & $\mathrm{q} 24.13$ & 210 & RP11-90D13 & AQ283623 & 110988 & 111165 \\
\hline 12 & $\mathrm{q} 24.33$ & 604 & RP11-146E8 & AQ372302 & 130310 & 130472 \\
\hline 13 & q21.1 & 213 & RP11-100C24 & AL353657 & 56600 & 56730 \\
\hline 13 & $\mathrm{q} 21.31$ & 2083 & RP11-151G10 & AQ377638 & 62481 & 62844 \\
\hline 13 & q31.1 & 216 & RP11-80N10 & AL136121 & 80203 & 80367 \\
\hline 13 & q31.1 & 218 & RP11-417I19 & AL162494 & 83615 & 83805 \\
\hline 14 & q11.1 & 605 & RP11-645B7 & AQ404284 & 18654 & 18834 \\
\hline 14 & q11.1 & 605 & RP11-831B15 & AQ818166 & 19273 & 19485 \\
\hline 14 & q12 & 225 & RP11-125A5 & AQ345964 & 28581 & 28769 \\
\hline 14 & q13.3 & 226 & RP11-26M6 & B84609 & 34404 & 34592 \\
\hline 14 & $\mathrm{q} 32.33$ & 232 & RP11-817G24 & AQ555246 & 104533 & 104760 \\
\hline 14 & q32 & 232 & RP11-141I7 & AQ484780 & 105830 & 105831 \\
\hline 15 & q11.1 & 233 & RP11-138C5 & AQ382833 & 19203 & 19367 \\
\hline 15 & q11.1 & 233 & RP11-2F9 & B63287 & 19790 & 19970 \\
\hline 15 & q12 & 236 & RP11-624A21 & AC068448 & 30139 & 30140 \\
\hline 15 & q13 & 236 & RP11-30N16 & AC021413 & 30213 & 30213 \\
\hline 15 & $\mathrm{q} 14.1$ & 241 & RP11-194H7 & AQ412869 & 32398 & 32459 \\
\hline 15 & q23 & 2084 & RP11-47G3 & AQ202180 & 69366 & 69367 \\
\hline 15 & q24.13 & 244 & RP11-500O23 & AZ301222 & 70607 & 70906 \\
\hline 15 & q24 & 245 & RP11-91O13 & AQ282541 & 80528 & 81083 \\
\hline 15 & $\mathrm{q} 25.2$ & 498 & RP11-246L14 & AQ480297 & 82722 & 82871 \\
\hline 15 & $\mathrm{q} 26.2$ & 247 & RP11-120N1 & AQ341084 & 95834 & 95937 \\
\hline 15 & $\mathrm{q} 26.3$ & 2085 & RP11-67J16 & AQ198995 & 99117 & 99292 \\
\hline 16 & p13.11 & 2086 & RP11-114I21 & AQ344890 & 15601 & 15768 \\
\hline 16 & p13.11 & 2087 & RP11-49G12 & AQ051855 & 16129 & 16286 \\
\hline 16 & p12 & 250 & RP11-94F6 & AC 133567 & 22200 & 30100 \\
\hline 16 & p11.2 & 2088 & RP11-159J3 & AQ372641 & 28012 & 28013 \\
\hline 16 & p11.2 & 2089 & RP11-2C24 & B48480 & 30747 & 30747 \\
\hline 16 & p11.2 & 252 & RP11-488I20 & AC007353 & 34289 & 34491 \\
\hline 16 & $\mathrm{p} 11.2$ & 251 & RP11-96K14 & AQ313535 & 32616 & 32617 \\
\hline 16 & q12.1 & 253 & RP11-419B13 & AQ553383 & 48813 & 48814 \\
\hline 16 & q22 & 612 & RP11-142E6 & AC133545 & 68662 & 68814 \\
\hline 16 & q24 & 255 & RP11-443M9 & AZ081877 & 84512 & 84607 \\
\hline 16 & $\mathrm{q} 24.3$ & 256 & RP1-191P24b & n.a. & Subtelo $16 q$ & Subtelo $16 q$ \\
\hline 17 & p12 & 614 & $\mathrm{RP} 11-721 \mathrm{~K} 1^{\mathrm{a}}$ & AC005411 & 13373 & 13538 \\
\hline 17 & $\mathrm{q} 12$ & 259 & RP11-430G19 & AQ552400 & 31949 & 32114 \\
\hline
\end{tabular}


Table I. Continued.

\begin{tabular}{|c|c|c|c|c|c|c|}
\hline Chr & Band & VL & BAC & $\mathrm{AC}$ & Start kb & End $\mathrm{kb}$ \\
\hline 17 & q12 & 259 & RP11-586K24 & AQ333429 & 33551 & 33602 \\
\hline 17 & $\mathrm{q} 21.2$ & 261 & RP11-29C11 & B87496 & 36749 & 36924 \\
\hline 17 & q21 & 263 & RP11-243L6 & AQ488523 & 41572 & 41734 \\
\hline 17 & $\mathrm{q} 23.3$ & 267 & RP11-89H15 & AC025362 & 59472 & 59627 \\
\hline 17 & $\mathrm{q} 24.2$ & 2093 & RP11-338N20 & AQ539594 & 62392 & 62393 \\
\hline 17 & $\mathrm{q} 25.3$ & 617 & RP11-762A9 & AQ454240 & 74989 & 75257 \\
\hline 17 & $\mathrm{q} 25.3$ & 270 & RP11-46E14 & AQ201029 & 75316 & 75477 \\
\hline 17 & $\mathrm{q} 25.3$ & 2094 & $\mathrm{RP} 11-334 \mathrm{C} 17^{\mathrm{a}}$ & AC015559 & 75616 & 75799 \\
\hline 18 & q12.3 & 274 & RP11-89M10 & AQ283397 & 37456 & 37620 \\
\hline 18 & q21 & 618 & RP11-742D12a & AC090311 & 42436 & 42622 \\
\hline 18 & q21.2 & 275 & RP11-160B24 & AQ377611 & 51861 & 52034 \\
\hline 18 & q22 & 619 & RP11-704G7a & AC021701 & 65892 & 66063 \\
\hline 19 & p13.2 & 530 & RP11-177L8 & AQ412496 & 8073 & 8277 \\
\hline 19 & p13.2 & 620 & RP11-79F15 & AQ284166 & 8714 & 8864 \\
\hline 19 & q13.31 & 1382 & RP11-313K22 ${ }^{b}$ & n.a. & n.a. & n.a. \\
\hline 19 & q13.31 & 280 & RP11-21J15 & AC013814 & 49726 & 49900 \\
\hline 19 & q13.33 & 2098 & RP11-264M8 & AQ486547 & 54767 & 54925 \\
\hline 19 & q13.33 & 2099 & RP11-369N17 & AQ531130 & 55229 & 55345 \\
\hline 19 & $\mathrm{q} 13.4$ & 621 & RP11-155P5 & AC026981 & 59909 & 59909 \\
\hline 20 & p12.1 & 283 & RP11-80N12 & AQ317837 & 16723 & 16896 \\
\hline 20 & q13.2 & 286 & RP4-749H19 & AL031674 & 54896 & 55076 \\
\hline 21 & p11.2 & 1394 & RP11-139O21 & AQ382339 & 10023 & 10187 \\
\hline 21 & q11.2 & 622 & RP11-1126H14 & AQ698240 & 13831 & 13832 \\
\hline 21 & $\mathrm{q} 21.1$ & 290 & RP11-49J9 & AQ053102 & 20982 & 21154 \\
\hline 21 & $\mathrm{q} 22.3$ & 293 & RP11-88N2 & AQ281525 & 43555 & 43770 \\
\hline 22 & q11.1 & 294 & RP11-134C5 & AQ386083 & 14759 & 14900 \\
\hline 22 & q11.21 & 627 & RP11-379N11 ${ }^{\mathbf{b}}$ & AQ532789 & n.a. & n.a. \\
\hline 22 & q11.2 & 625 & RP11-775G6 & AQ514830 & 19859 & 19860 \\
\hline 22 & q11.22 & 2011 & RP11-359L2 & AQ541478 & 21416 & 21417 \\
\hline 22 & q11.23 & 296 & RP11-76E8 & AQ265558 & 22800 & 22995 \\
\hline 22 & q11.23 & 2014 & RP11-157B2 & AQ374326 & 23928 & 24093 \\
\hline 22 & q12.1 & 2104 & RP11-259P1 & AQ483010 & 24843 & 25024 \\
\hline 22 & q12.3 & 299 & RP11-89D12 & AQ285741 & 34601 & 34671 \\
\hline$X$ & $\mathrm{p} 22.33$ & 628 & RP11-23N11 & AQ014327 & 3862 & 3863 \\
\hline $\mathbf{X}$ & p22.31 & 553 & RP11-111F17 & AQ341345 & 9318 & 9461 \\
\hline $\mathrm{X}$ & $\mathrm{p} 21.31$ & 303 & RP6-27C10 & AL031803 & 29091 & 29143 \\
\hline $\mathrm{X}$ & p21.1 & 304 & RP4-769D20 & AL031643 & 33118 & 33253 \\
\hline $\mathbf{X}$ & p11.1 & 305 & RP3-323P24 & AL022157 & 57113 & 57194 \\
\hline $\mathbf{X}$ & q13.2 & 306 & RP13-36G14 & AL139400 & 73105 & 73208 \\
\hline $\mathbf{X}$ & q21.1 & 309 & RP1-223D17 & AL121882 & 82645 & 82778 \\
\hline$X$ & $\mathrm{q} 21.32$ & 311 & RP11-156J23 & AL158053 & 92133 & 92310 \\
\hline $\mathbf{X}$ & $\mathrm{q} 22.3$ & 313 & RP1-75H8 & AL158821 & 106053 & 106230 \\
\hline $\mathrm{X}$ & $\mathrm{q} 25$ & 314 & RP6-64P14 & AL109800 & 121879 & 122069 \\
\hline$X$ & q26 & 1449 & RP1-119E23 & Z99570 & 132871 & 132937 \\
\hline$X$ & q26 & 2106 & RP11-11I8 & B71885 & 134067 & 134250 \\
\hline $\mathrm{Y}$ & p11.2 & 2107 & RP11-35D7 & AQ045270 & 6265 & 6266 \\
\hline Y & p11.2 & 636 & RP11-731O16 & AQ453742 & 9927 & 9928 \\
\hline Y & q11.2 & 640 & RP11-135P22 & AQ382241 & 22770 & 22770 \\
\hline Y & q11.221 & 2108 & RP11-268K13a & AC022848 & 18627 & 18791 \\
\hline Y & q11.223 & 2110 & RP11-355K9 & AQ533417 & 23406 & 23406 \\
\hline $\mathrm{Y}$ & q11.223 & 641 & RP11-458F2 & AQ584194 & 23696 & 23861 \\
\hline Y & q11.223 & 2113 & RP11-69C24 & AQ236655 & 24578 & 24726 \\
\hline
\end{tabular}

Chr, chromosome; Band, cytogenetic band; VL, variation locus; BAC, AC-accession code; Start kb and End kb, start and end of the BAC clone on the chromosome. The BAC clones that showed a polymorphism on the tested female subject are indicated in bold print. ${ }^{\mathrm{a}} \mathrm{Only}$ available on NCBI 35 ; bonly available on the database of genomic variants. 


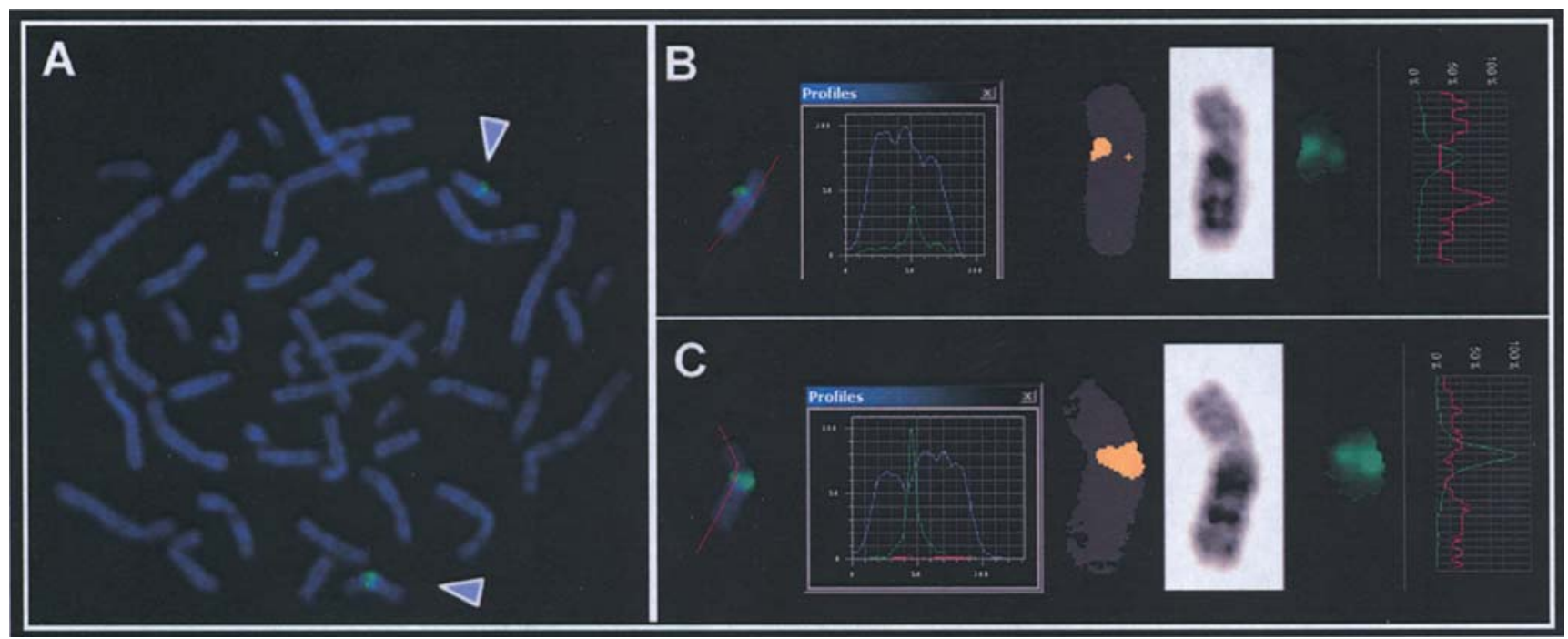

Figure 2. Example of a single pod-FISH hybridization of BAC clone RP11-488I20 (16p11.2). The signal intensity difference between the homologous chromosomes were easily detected by the naked eye on the metaphase spread (A) or by analyzing fluorochrome profiles (B, C). All examined metaphase spreads show the weaker fluorescence signal on chromosome 16 with the smaller heterochromatin block and the stronger one on the homologous chromosome with qh+ variant.

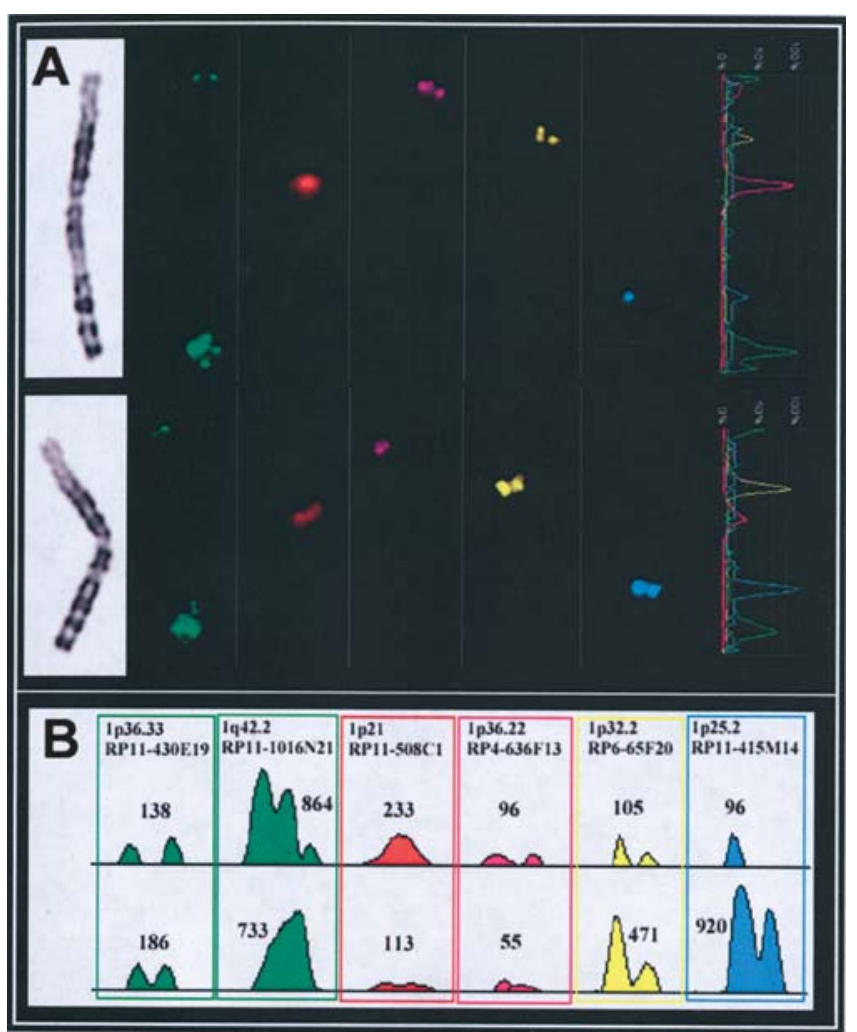

Figure 3. pod-FISH probe set for chromosome 1 showing strong signal intensity differences for the Spectrum Orange-, Cy5- and DEAC-labeled BAC clone. This was analyzed by fluorochrome profiles (A, left to right: inverted DAPI, FITC, Spectrum Orange, Texas Red, Cy5, and DEAC) and measured by SCION software (B). Values are provided for each fluorochrome channel; homologous chromosomes are placed one below the other.

partially or completely lost. In molecular cytogenetics a loss, e.g. in microdeletion syndromes, is unambiguously detected, while a small gain of copy number, like in microduplication syndromes (23), is much harder to distinguish. Since there are no studies available on LCV/CNP frequency in the general population only regions were taken into account, that were observed in more than one person or study. Additionally, these regions needed to have a size $>150 \mathrm{~kb}$ in order to be detectable in FISH. According to these requirements we selected 225 BAC clones from the NCBI database (http://www.ncbi.nlm. nih.gov/) as listed in Table I.

All probes were initially tested on chromosomes from one clinically healthy person. Here, the overall frequency of podFISH detectable polymorphisms was 29.3\%; 66 of 225 regions showed a complete loss or a microscopically detectable decrease in fluorescence intensity in one of the two homologous chromosome (BAC clones in bold print; Table I).

The evaluation of pod-FISH probes is able to be performed in several ways: i) by naked eye using a fluorescence microscope (Fig. 2A), ii) by analyzing fluorescence profiles with an appropriate software (Fig. 2B and C; Fig. 3A) and iii) by measuring signal intensity and area with a software that was previously proven suitable for measuring FISH intensity signal. For our purposes, the freeware SCION (http:www. scioncorp.com) was applied (25) (Fig. 3B).

In order to observe real signal intensity differences caused by LCV/CNP polymorphisms and not variations of the FISH method itself, we analyzed 10-25 metaphase spreads per hybridization to obtain a semi-statistical mean value.

Creation and evaluation of pod-FISH sets. As it is useful to work with more than one polymorphic BAC probe simultaneously, chromosome-specific pod-FISH sets based on 5 different fluorochromes were created. For larger chromosomes such as 1, 2, 3, 4, 6, 9 and $\mathrm{X}$, it was more convenient to generate chromosome arm-specific pod-FISH sets for an easier analysis and to prevent double labeling. A corresponding overview of all 31 human pod-FISH sets is provided in Fig. 5. 


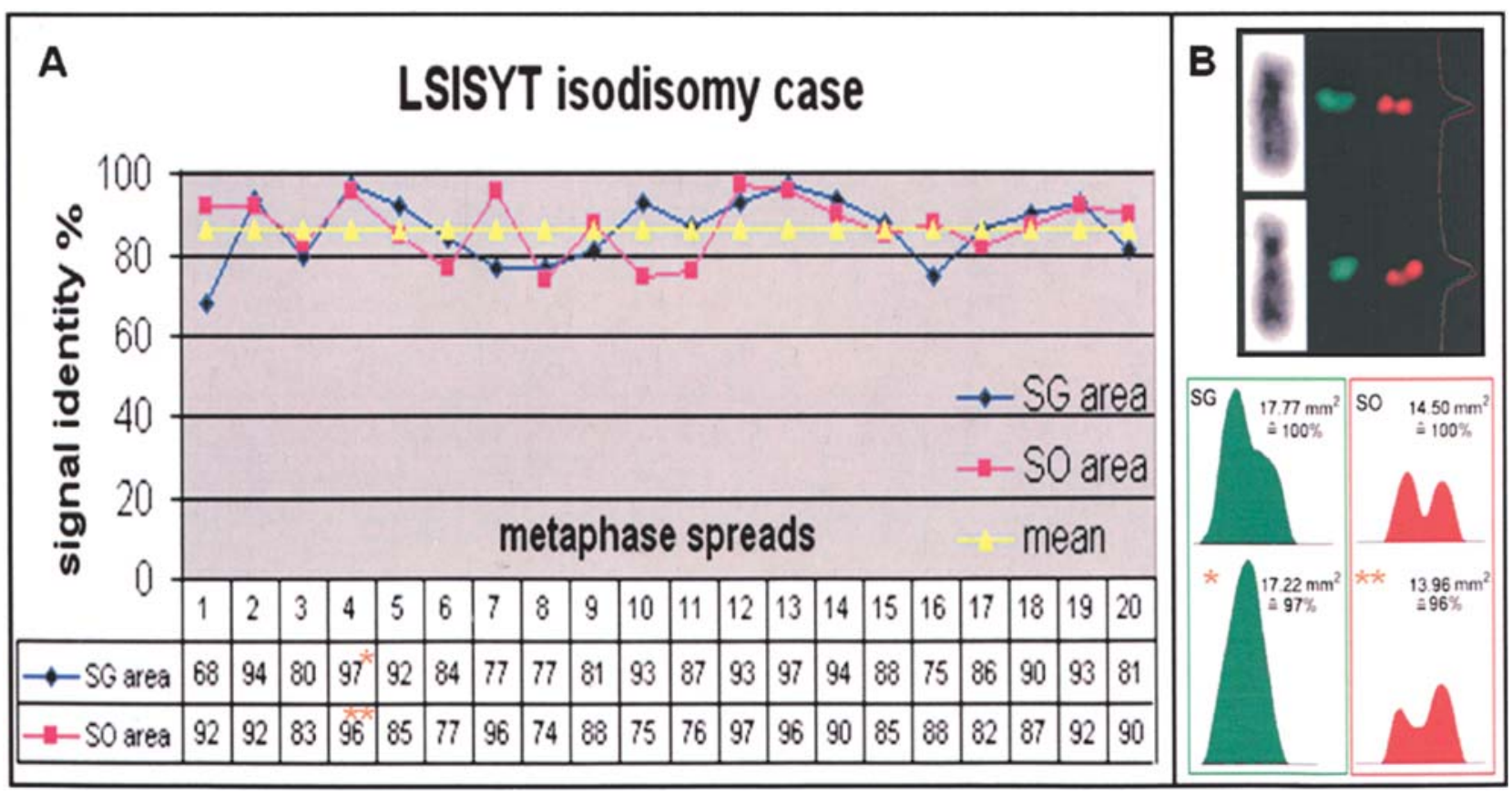

Figure 4. Cut-off assignment for FISH signal identity of non-polymorphic regions on homologous chromosomes. The example shows the values and curves for the LSISYT probe in Spectrum Orange (SO) and Spectrum Green (SG) on 20 metaphase spreads of a clinical case with a mosaic of normal and complete isodisomy. For the analysis, only isodisomic cells were chosen (A). The signal area was measured by SCION (B). The stronger signal was set to $100 \%$. The mean identity between the signal areas of both homologous chromosomes was $86.7 \%$. The detailed measuring is demonstrated for metaphase number 4 (*) in picture B. Fluorescent microscopic undistinguishable homologous chromosomes (top) were measured by SCION (bottom) and compared as previously described.

To test the reliability and the reproducibility of the podFISH approach in distinguishing homologous chromosomes, two carriers of a heterochromatic variant on one of the three chromosomes 1,9 or 16 each were selected. pod-FISH sets as well as single probes for the corresponding three chromosomes were applied. Thus, the chromosome-specific podFISH intensity pattern could be correlated with the cytogenetic distinguishable homologous chromosomes. We analyzed 1025 metaphase spreads per hybridization and found the identical, distinguishable hybridization pattern on the chromosomes with and without the heteromorphism, respectively (Fig. 2).

In order to obtain a cut-off value for differentiation between real polymorphisms and methodically caused variations within one hybridization we selected two commercially available probes (LSI SYT, 18q11.2, Abbott and LSI ABL, Abbott) and two BAC clones (RP11-358M9 and RP11175A7) that were not located in known CNP/LCV regions. Twenty metaphase spreads per hybridization were analyzed in lymphocytes from two clinically healthy persons and one in bone marrow from a leukemia patient. Additionally, lymphocytes of a rare case of complete paternal isodisomy in $97 \%$ of blood cells was used with the above mentioned probes labeled in two different fluorochromes (Spectrum Orange or Spectrum Green) to uncover the influences of the labeling on the FISH result. In fact no microscopically visible differences were detected between the homologous chromosomes in all analyzed metaphase spreads applying the two different fluorochromes in all probands. Nevertheless, employing the sensitive SCION software we were able to measure the intensity and area of the signals on both homologous chromosomes; the more intense signal was defined as $100 \%$. The mean value of the second signal relative to the reference signal was not $<73-90 \%$ for both commercially available probes and not $<74-80 \%$ for the BAC probes (Fig. 4). Therefore, we concluded that the normal variation of the FISH method for signal identity on both homologous chromosomes for a non-polymorphic region is between 10 and $36 \%$. Thus, if in the polymorphic region the difference was $>36 \%$, a real polymorphism distinguishing both homologous chromosomes was suggested. The aforementioned experiments also demonstrated the high sensitivity of the SCION software to measure differences not visible to the naked eye (Fig. 4).

pod-FISH in UPD analysis. Imprinting of genes can cause specific syndromes when certain chromosomes are inherited from one parent only. Associated with a clinical phenotype are paternal UPD 6, maternal UPD 7, paternal UPD 11, maternal UPD 16 and maternal as well as paternal UPD 14 and 15 (reviewed in ref. 26). As described above, UPD can be investigated by microsatellite analysis of the parents and the child to determine if one parent transferred both alleles and or homologous chromosomes to the child. Therefore, we applied the pod-FISH set for chromosome 15 to a UPD case, unaware of which parent the chromosomes were inherited from, and compared the pod-FISH pattern of the parents and the child. We were able to confirm the molecular genetic microsatellite results on chromosomes investigated with podFISH. An example of a UPD 15 confirmation is shown in Fig. 7. It was clearly demonstrated that none of the signal patterns of the father were found in the child which could 


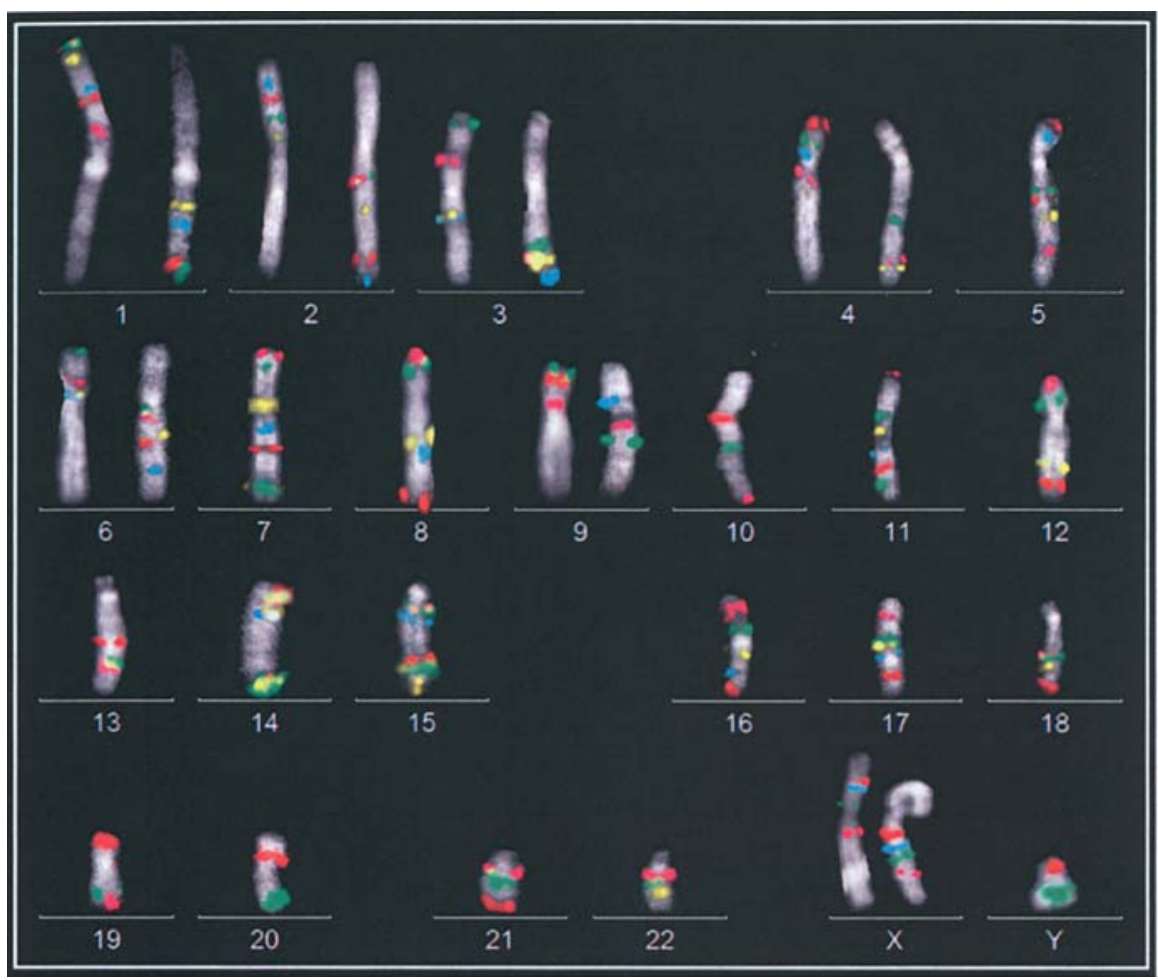

Figure 5. Overview of all 31 available chromosome-specific pod-FISH sets (independent hybridizations). For a better analysis and to prevent double labeling, we created chromosome arm-specific sets for chromosomes 1,2,3,4,6,9 and X.

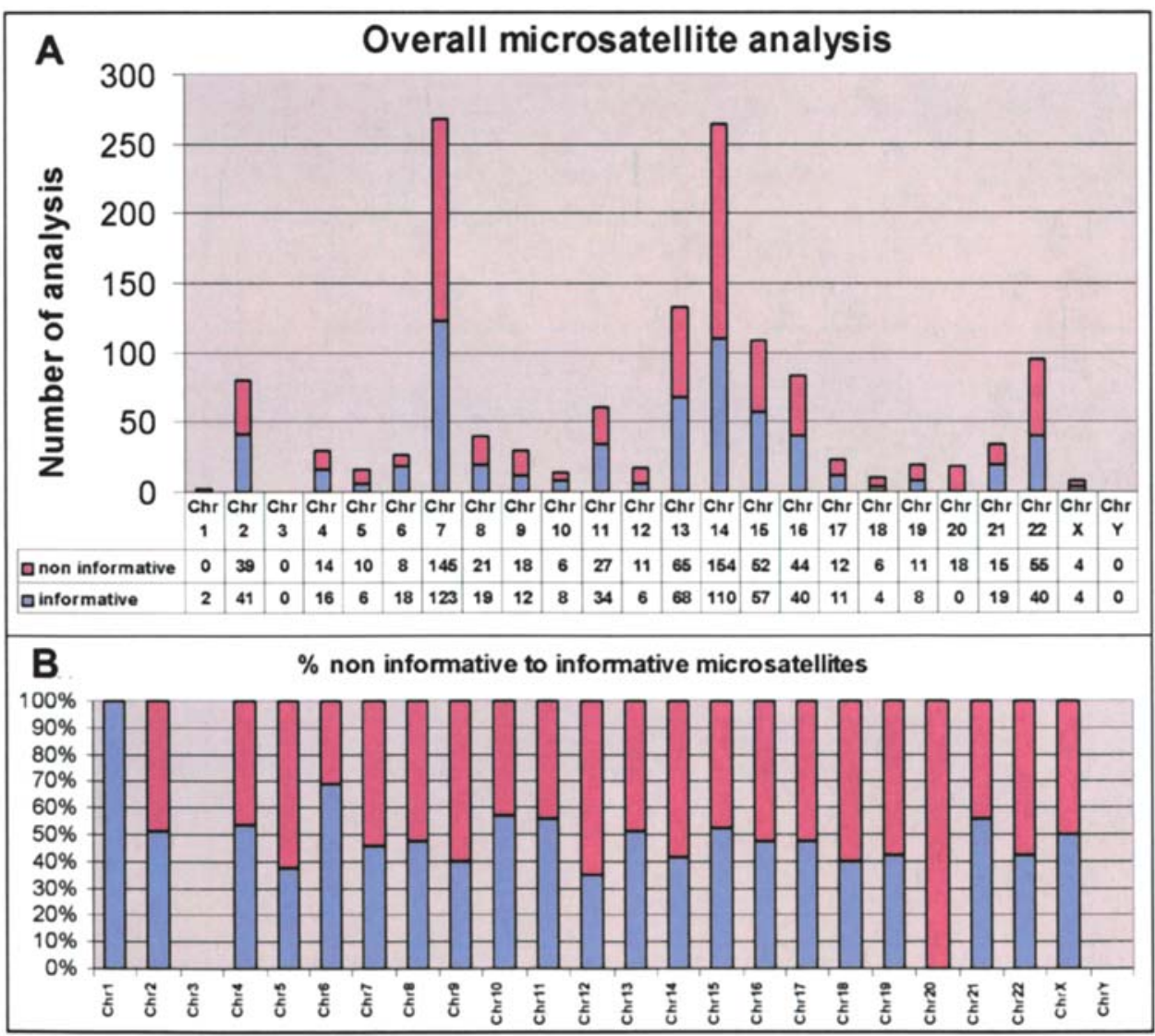

Figure 6. UPD statistics from 1545 single microsatellites showing non-informative (red) and informative (blue) results (A) in respect to the chromosomes analyzed. The overall mean value for informative situations was 47.1 versus $52.9 \%$ for non-informative situations that varied in different chromosomes depending on the number of analyzed microsatellites per chromosome (B). 
Table II. Summarized results of informative situations in relation to the number of used BACs (i./n. BACs) in the 5 subjects tested for chromosomes 1 to $6 .^{\text {a }}$

\begin{tabular}{|c|c|c|c|c|c|c|}
\hline Test person & $\begin{array}{c}\text { Chr } 1 \\
\text { i./n. BACs }\end{array}$ & $\begin{array}{c}\text { Chr } 2 \\
\text { i./n. BACs }\end{array}$ & $\begin{array}{c}\text { Chr } 3 \\
\text { i./n. BACs }\end{array}$ & $\begin{array}{c}\text { Chr } 4 \\
\text { i./n. BACs }\end{array}$ & $\begin{array}{c}\text { Chr } 5 \\
\text { i./n. BACs }\end{array}$ & $\begin{array}{c}\text { Chr } 6 \\
\text { i./n. BACs }\end{array}$ \\
\hline 1 & $0 / 12$ & $2 / 10$ & $4 / 8$ & $1 / 7$ & $1 / 6$ & $0 / 9$ \\
\hline 2 & $1 / 12$ & $0 / 10$ & $0 / 8$ & $0 / 7$ & $3 / 6$ & $2 / 9$ \\
\hline 3 & $2 / 12$ & $1 / 10$ & $1 / 8$ & $1 / 7$ & $0 / 6$ & $0 / 9$ \\
\hline 4 & $0 / 12$ & $0 / 10$ & $2 / 8$ & $1 / 7$ & $1 / 6$ & $0 / 9$ \\
\hline 5 & $1 / 12$ & $0 / 10$ & $0 / 8$ & $0 / 7$ & $0 / 6$ & $0 / 9$ \\
\hline $\begin{array}{l}\text { Differentiation } \\
\text { possible in }\end{array}$ & $3 / 5$ cases & $2 / 5$ cases & $3 / 5$ cases & $3 / 5$ cases & $3 / 5$ cases & $1 / 5$ cases \\
\hline
\end{tabular}

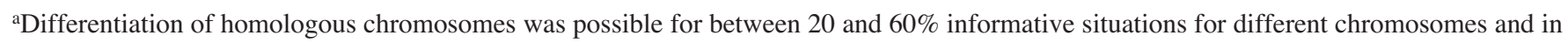
$50 \%$ of all chromosome-specific pod-FISH sets.

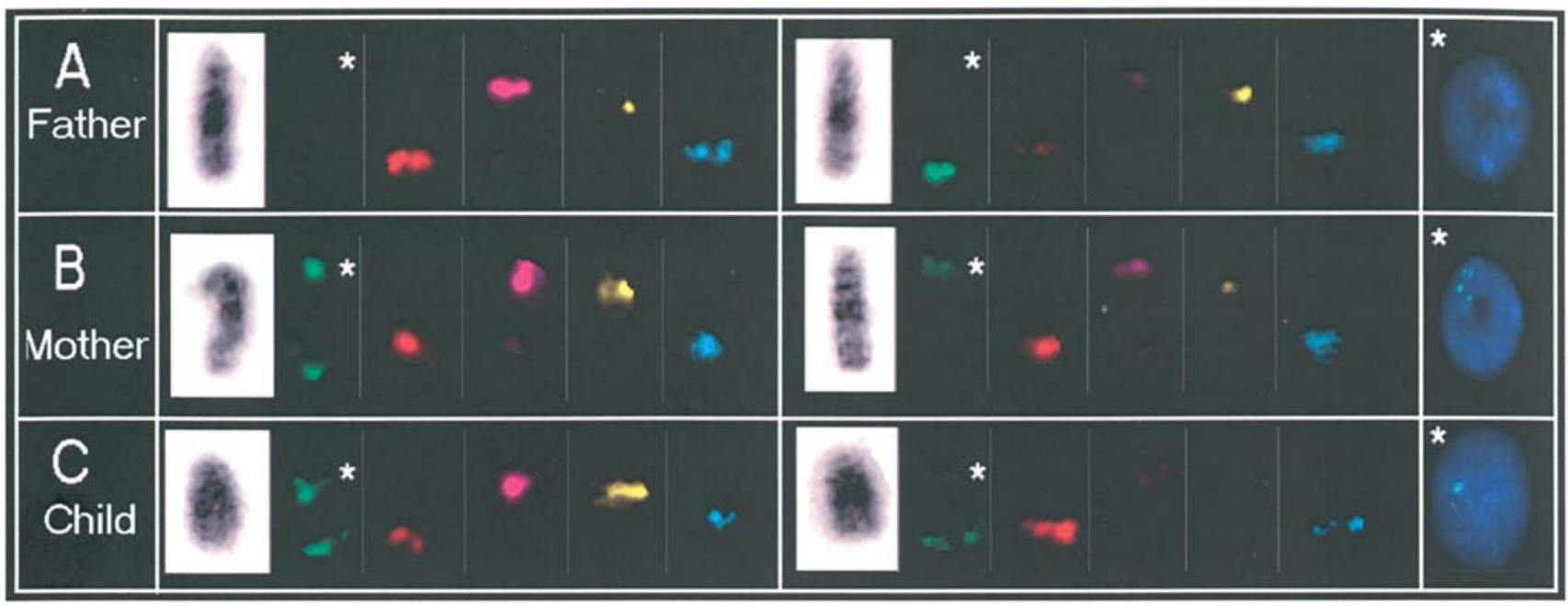

Figure 7. pod-FISH confirmation of a maternal heterodisomy 15. Comparison of the signal patterns from homologous chromosomes 15 (left to right: inverted DAPI, FITC, Spectrum Orange, Texas Red, Cy5 and DEAC) of the father (A), the mother (B), and the child (C). None of the fluorochrome compositions on both chromosomes 15 from the father fit those of the child. Yet, both fluorochrome patterns of the mother fit those of the child. *The spectrum green-labeled BAC clone RP11-138C5 in 15q11.1 that exemplifies the feasibility for interphase analysis (rightmost panels).

also be interpreted as non-paternity, but both pod-FISH patterns of the mother fit exactly to the child confirming the previously found maternal heterodisomy 15 in the child. Moreover, UPD was previously determined by microsatellite analysis (data not shown).

pod-FISH compared to microsatellite analysis. As in the well-established microsatellite analysis, the use of pod-FISH similarly can result in so-called 'non-informative' findings. This shows that the same polymorphic region on both homologous chromosomes can have the identical size and therefore are undistinguishable by the applied approach. However, there are no reports or statistics concerning the genome-wide heterozygosity frequency and/or informative situation in UPD testing by microsatellites.

In order to obtain such a dataset with which to compare the rate of informativeness of pod-FISH, the results of 177 UPD analyses by microsatellite performed in Caucasians were collected in Fig. 6. Data for all chromosomes were available apart from chromosomes 3 and Y. In summary, 1545 microsatellite PCRs on the basis of 299 different microsatellite probes resulted in $727(47.1 \%)$ non-informative versus $818(52.9 \%)$ informative findings (Fig. 6) that allowed a distinctive differentiation of alleles in a parent-child-trio. Therefore, approximately every second microsatellite leads to an informative situation. After evaluation of five healthy control persons for pod-FISH sets of chromosomes 1 to 6 (in summary 53 different probes in 30 experiments) a comparable rate of informativeness was observed in 20-60\% of the analyzed chromosomes. In $50 \%$ of all chromosomespecific pod-FISH sets, signal intensity differences were observed (Table II). Nevertheless, a higher degree of informative LCV/CNP loci is needed to result in less noninformative situations. This can be achieved by inclusion of more BAC probes specific for other polymorphic loci in chromosome-specific pod-FISH sets. Information enabling this will be available by published CNP/LCV as more and more studies are performed studying individuals for allele 
frequency estimations and de novo mutation rates. Currently we are working on a genome-wide high frequency CNP/LCV set with population incidences $>20 \%$ based mainly on recent reports from the 270 Hap map individuals $(19,20)$.

Future applications. pod-FISH opens a wide range of possibilites for new fields in research and diagnostics. In this study we made a first approach towards the evaluation of pod-FISH and new diagnostic areas like UPD analysis on metaphase spreads. Potential areas of use include the exclusion of maternal contamination in prenatal diagnosis, detection of chromosome homozygosity processes (e.g. in tumor genesis or aging), the determination of the origin of aberrant chromosomes, the determination of cell mixtures or mosaics (e.g. in clinical monitoring of leukemia patients), proof of paternity by chromosomes and following single chromosomes in generations by pod-FISH.

Microsatellite or SNP approaches are the method of choice to distinguish genomic DNA on a molecular level, but they are unable to determine which allele belongs to which homologous chromosome. Therefore, pod-FISH is the only method able to answer these questions. Furthermore, for molecular approaches, DNA is isolated from a number of cells that can differ in their genomic content, e.g. in cases of mosaicism. In contrast, pod-FISH analyzes single cells where mosaicism can easily be uncovered and interpreted. This can play a significant role, for instance, in AML cases where segmental UPDs were reported for chromosomes 6, 9, 10, 11, $12,13,15,16,19$ and 21 that could only be detected by SNP arrays when the tumor cell fraction was $>70 \%$ (27-31). Inferentially, all AMLs with a lower portion of tumor cells are not analyzable by this method although these epigenetic changes can importantly be used as tumor markers.

In conclusion, pod-FISH is the first cytogenetic method to distinguish homologous chromosomes or sequences based on submicroscopic DNA polymorphisms. The main advantage of this new technique is its performance on single cells. Furthermore, it is suitable for every chromosome. With an expected growing number of reported CNP/LCV a more dense genome-wide coverage will be achieved and more information will be available concerning single frequencies.

\section{Acknowledgements}

This study was supported, in part, by a grant from the University of Jena, the Deutsche Krebshilfe (70-3125-Li1), the INTAS (AISbl 03-51-4060), the IZKF Jena (Start-up S16), the DFG (436 ARM 17/5/06), IZKF together with the TMWFK (TP 3.7 and B307-04004), the Ernst-Abbe-Stiftung, the Stiftung Leukämie, and the Evangelische Studienwerk e.V. Villigst.

\section{References}

1. Lee C: Vive la difference! Nat Genet 37: 660-661, 2005.

2. Schlötterer C: The evolution of molecular markers - just a matter of fashion? Nat Rev Genet 5: 63-69, 2004.

3. Liehr T, Nietzel A, Starke H, Heller A, Weise A, Kuechler A, Senger G, Ebner S, Martin T, Stumm M, Wegner R, Tonnies H, Hoppe C, Claussen U and von Eggeling F: Characterization of small marker chromosomes (SMC) by recently developed molecular cytogenetic approaches. J Assoc Genet Technol 29: 5-10, 2003.
4. Nietzel A, Albrecht B, Starke H, Heller A, Gillessen-Kaesbach G, Claussen U and Liehr T: Partial hexasomy 15pter-->15q13 including SNRPN and D15S10: first molecular cytogenetically proven case report. J Med Genet 40: e28, 2003.

5. Müller H, Klinger HP and Glasser M: Chromosome polymorphism in a human newborn population. II. Potentials of polymorphic chromosome variants for characterizing the idiogram of an individual. Cytogenet Cell Genet 15: 239-255, 1975.

6. ISCN: An International System for Human Cytogenetic Nomenclature. Shaffer LG and Tommerup N (eds). S. Karger, Basel, 2005.

7. Gardner RGM and Sutherland GR: Oxford Monographs on Medical Genetics No. 46: Chromosome Abnormalities and Genetic Counselling. 3rd edition, Oxford University Press, Oxford, 2004.

8. Liehr T, Ziegler M, Starke H, Heller A, Kuechler A, Kittner G, Beensen V, Seidel J, Hassler H, Musebeck J and Claussen U: Conspicuous GTG-banding results of the centromere-near region can be caused by alphoid DNA heteromorphism. Clin Genet 64: 166-167, 2003.

9. Iafrate AJ, Feuk L, Rivera MN, Listewnik ML, Donahoe PK, Qi Y, Scherer SW and Lee C: Detection of large-scale variation in the human genome. Nat Genet 36: 949-951, 2004.

10. Sebat J, Lakshmi B, Troge J, Alexander J, Young J, Lundin P, Månér S, Massa H, Walker M, Chi M, Navin N, Lucito R, Healy J, Hicks J, Ye K, Reiner A, Gilliam TC, Trask B, Patterson N, Zetterberg A and Wigler M: Large-scale copy number polymorphism in the human genome. Science 23: 525-528, 2004.

11. Shaw-Smith C, Redon R, Rickman L, Rio M, Willatt L, Fiegler H, Firth H, Sanlaville D, Winter R, Colleaux L, Bobrow M and Carter NP: Microarray based comparative genomic hybridisation (array-CGH) detects submicroscopic chromosomal deletions and duplications in patients with learning disability/ mental retardation and dysmorphic features. J Med Genet 41: 241-248, 2004.

12. Sharp AJ, Locke DP, McGrath SD, Cheng Z, Bailey JA, Vallente RU, Pertz LM, Clark RA, Schwartz S, Segraves R, Oseroff VV, Albertson DG, Pinkel D and Eichler EE: Segmental duplications and copy-number variation in the human genome. Am J Hum Genet 77: 78-88, 2005.

13. Tuzun E, Sharp AJ, Bailey JA, Kaul R, Morrison VA, Pertz LM, Haugen E, Hayden H, Albertson D, Pinkel D, Olson MV and Eichler EE: Fine-scale structural variation of the human genome. Nat Genet 37: 727-732, 2005.

14. de Vries BB, Pfundt R, Leisink M, Koolen DA, Vissers LE, Janssen IM, Reijmersdal S, Nillesen WM, Huys EH, Leeuw N, Smeets D, Sistermans EA, Feuth T, van Ravenswaaij-Arts CM, van Kessel AG, Schoenmakers EF, Brunner HG and Veltman JA: Diagnostic genome profiling in mental retardation. Am J Hum Genet 77: 606-616, 2005.

15. Feuk L, MacDonald JR, Tang T, Carson AR, Li M, Rao G, Khaja R and Scherer SW: Discovery of human inversion polymorphisms by comparative analysis of human and chimpanzee DNA sequence assemblies. PLoS Genet 1: 56, 2005.

16. Schoumans J, Ruivenkamp C, Holmberg E, Kyllerman M, Anderlid BM and Nordenskjold M: Detection of chromosomal imbalances in children with idiopathic mental retardation by array-based comparative genomic hybridisation (array-CGH). J Med Genet 42: 699-705, 2005.

17. Conrad DF, Andrews TD, Carter NP, Hurles ME and Pritchard JK: A high-resolution survey of deletion polymorphism in the human genome. Nat Genet 38: 75-81, 2006.

18. Hinds DA, Kloek AP, Jen M, Chen X and Frazer KA: Common deletions and SNPs are in linkage disequilibrium in the human genome Nat Genet 38: 82-85, 2006.

19. Locke DP, Sharp AJ, McCarroll SA, McGrath SD, Newman TL, Cheng Z, Schwartz S, Albertson DG, Pinkel D, Altshuler DM and Eichler EE: Linkage disequilibrium and heritability of copy-number polymorphisms within duplicated regions of the human genome. Am J Hum Genet 79: 275-290, 2006.

20. Redon R, Ishikawa S, Fitch KR, Feuk L, Perry GH, Andrews TD, Fieglerm H, Shapero MH, Carson AR, Chen W, Cho EK, Dallaire S, Freeman JL, Gonzalez JR, Gratacos M, Huang J, Kalaitzopoulos D, Komura D, MacDonald JR, Marshall CR, Mei R, Montgomery L, Nishimura K, Okamura K, Shen F, Somerville MJ, Tchinda J, Valsesia A, Woodwark C, Yang F, Zhang J, Zerjal T, Zhang J, Armengol L, Conrad DF, Estivill X, Tyler-Smith C, Carter NP, Aburatani H, Lee C, Jones KW, Scherer SW and Hurles ME: Global variation in copy number in the human genome. Nature 444: 444-454, 2006. 
21. Verma RS and Babu A: Human Chromosomes - Manual of Basic Techniques. Pergamon Press, New York, 1989.

22. Liehr T, Heller A, Starke H, Rubtsov N, Trifonov V, Mrasek K, Weise A, Kuechler A and Claussen U: Microdissection based high resolution multicolor banding for all 24 human chromosomes. Int J Mol Med 9: 335-339, 2002.

23. Liehr T, Thoma K, Kammler K, Gehring C, Ekici A, Bathke KD, Grehl H and Rautenstrauss B: Direct preparation of uncultured EDTA-treated or heparinized blood for interphase FISH analysis. Appl Cytogenet 21: 185-188, 1995.

24. Salafsky IS, MacGregor SN, Claussen U and von Eggeling F: Maternal UPD 20 in an infant from a pregnancy with mosaic trisomy 20. Prenat Diagn 21: 860-863, 2001.

25. Iourov IY, Soloviev IV, Vorsanova SG, Monakhov VV and Yurov YB: An approach for quantitative assessment of fluorescence in situ hybridization (FISH) signals for applied human molecular cytogenetics. J Histochem Cytochem 53: 401-408, 2005.

26. Kotzot D and Utermann G: Uniparental disomy (UPD) other than 15: phenotypes and bibliography updated. Am J Med Genet 136: 287-305, 2005.

27. Fitzgibbon J, Smith LL, Raghavan M, Smith ML, Debernardi S, Skoulakis S, Lillington D, Lister TA and Young BD: Association between acquired uniparental disomy and homozygous gene mutation in acute myeloid leukemias. Cancer Res 65: 9152-9154, 2005.
28. Gorletta TA, Gasparini P, D'Elios MM, Trubia M, Pelicci PG and Di Fiore PP: Frequent loss of heterozygosity without loss of genetic material in acute myeloid leukemia with a normal karyotype. Genes Chromosomes Cancer 44: 334-337, 2005.

29. Lu YJ, Yang J, Noel E, Skoulakis S, Chaplin T, Raghavan M, Purkis T, McIntyre A, Kudahetti SC, Naase M, Berney D, Shipley J, Oliver RT and Young BD: Association between large-scale genomic homozygosity without chromosomal loss and nonseminomatous germ cell tumor development. Cancer Res 65: 9137-9141, 2005.

30. Raghavan M, Lillington DM, Skoulakis S, Debernardi S, Chaplin T, Foot NJ, Lister TA and Young BD: Genome-wide single nucleotide polymorphism analysis reveals frequent partial uniparental disomy due to somatic recombination in acute myeloid leukemias. Cancer Res 65: 375-378, 2005.

31. Bungaro S, Raghavan M, Dell'Oro MG, Paolucci P, Young BD, Biondi A and Cazzaniga G: Assessment of submicroscopic genetic lesions by single nucleotide polymorphism arrays in a child with acute myeloid leukemia and FLT3-internal tandem duplication. Haematologica 91: 998-1000, 2006. 